\title{
Editorial
}

\section{A Nova Seção da Revista de Administração Contemporânea: Artigos-Tutoriais}

The New Section of the Journal of Contemporary Administration: Tutorial Articles

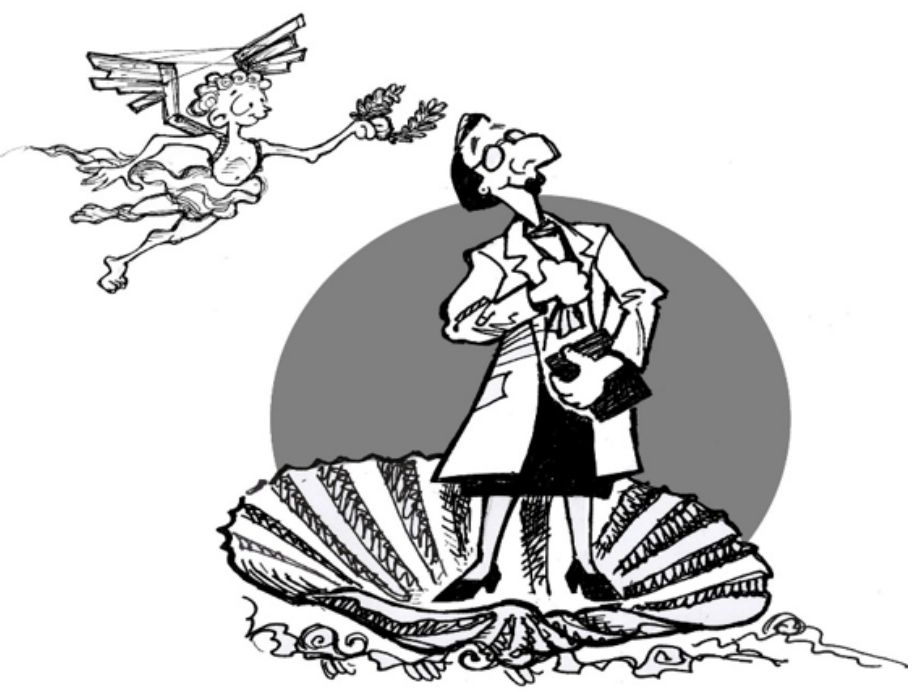

Figura 1. Ilustração de John R. McKiernan. Fonte: http://whyopenresearch.org/

\section{O QUE É UM ARTIGO-TUTORIAL?}

A Revista de Administração Contemporânea (RAC), desde o início de 2020, passou a aceitar Artigos-Tutoriais. Para incentivar a submissão de Artigos-Tutoriais, a RAC criou duas chamadas para trabalhos: Martins (2019) e Lanka, Lanka, Rostron e Singh (2019). Essas duas chamadas, ainda em

\footnotetext{
${ }^{1}$ Pontifícia Universidade Católica do Rio de Janeiro, IAG - Escola de Negócios, Rio de Janeiro, RJ, Brasil.

${ }^{2}$ Editor Associado de Artigos-Tutoriais e Dados Abertos da RAC - Revista de Administração Contemporânea.

${ }^{3}$ Fundação Getulio Vargas, Escola de Administração de Empresas de São Paulo, São Paulo, SP, Brasil.

${ }^{4}$ Editor-chefe da RAC - Revista de Administração Contemporânea.
}

aberto na data deste Editorial, oficializaram o início de uma nova seção da revista, que já nasce cercada por grande expectativa de tornar-se, em um futuro próximo, fonte importante de consulta aos métodos relevantes à pesquisa empírica em Administração.

Desde a publicação das chamadas para trabalhos, felizmente, diversos colaboradores da RAC pediram mais detalhamento acerca da estrutura

Como citar: Martins, H. C., \& Mendes-da-Silva, W. (2020). The new section of the Journal of Contemporary Administration: Tutorial articles. Revista de Administração Contemporânea, 24(3), 275-282. https://doi.org/10.1590/1982-7849rac2020200044 
e organização de Artigos-Tutoriais e acerca do processo editorial (mais especificamente, acerca do processo de revisão pelos pares) envolvido. Um dos objetivos desse editorial é, portanto, esclarecer questionamentos dirigidos ao Corpo Editorial e facilitar a comunicação daquilo que a RAC espera publicar nessa nova seção. Espera-se, com isso, aumentar a transparência acerca dessa nova seção e guiar as futuras submissões feitas à revista.

De acordo com o periódico acadêmico internacional Micron - The International Research and Review Journal for Microscopy, ArtigosTutoriais "deal with a particular area or technique of microscopy, making it understandable to beginners or experts and concentrating on practical aspects of implementation" (Elsevier, n.d., online). A RAC vem assumindo essa definição.
Para a RAC, os Artigos-Tutoriais deverão abordar e auxiliar pesquisadores, iniciantes e experientes, a executar e implementar, de forma objetiva, aspectos e técnicas empíricas relevantes a uma pesquisa científica em Administração. ArtigosTutoriais deverão escolher e lidar com uma técnica específica e facilitar a aplicação prática dessa técnica.

Naturalmente, uma parte importante da criação de um tutorial é abordar aspectos relacionados à implementação da técnica escolhida, que deverá ser relevante à pesquisa, quantitativa ou qualitativa, em Administração. Com isso, nosso objetivo é criar uma fonte de consulta acerca de práticas empíricas relevantes para nosso campo de pesquisa. Uma estrutura ideal, porém não obrigatória, de um Artigo-Tutorial está descrita na Figura 2.

1. Breve introdução e aplicabilidade do modelo/método: nessa etapa, os autores apresentam o potencial problema (da técnica ou do método empírico) enfrentado pelo pesquisador-leitor e ressaltam as consequências empíricas da falta de correções ao problema.

2. Discussão da teoria correlata e proposição de uma estrutura e de um problema de pesquisa empírico coerente com o modelo/método escolhido: nessa etapa, os autores apresentam oportunamente aspectos teóricos relevantes à aplicação da técnica ou do método estudado e discutem brevemente um problema de pesquisa coerente com a utilização dessa técnica. Acreditamos que essa etapa é importante para ilustrar e contextualizar o problema empírico enfrentado pelo pesquisadorleitor, de forma que incentivamos sua inclusão no Artigo-Tutorial a ser submetido.

3. Discussão/ilustração dos dados utilizados: nessa etapa, os autores são convidados a apresentar todos os desdobramentos e aspectos relacionados aos dados empíricos utilizados. Ressaltamos que desde 2018 a RAC vem gradativamente incrementando o apoio a diferentes iniciativas relacionadas ao Movimento de Ciência Aberta, conforme temos continuamente detalhado em nossos editoriais, com a intenção de fornecer à comunidade elementos dirigidos ao amadurecimento à assimilação das práticas mais modernas em termos de processo editorial e disseminação de conhecimento (Mendes-Da-Silva, 2018a). Dessa forma, é importante os autores utilizarem dados que possam ser disponibilizados virtualmente de forma livre e sem restrições ao pesquisador-leitor. Analogamente, é importante que os autores apresentem e disponibilizem os códigos, scripts ou demais arquivos de estimação empírica utilizada no pacote estatístico de forma livre e sem restrições ao pesquisador-leitor.

4. Elucidação e explicação das etapas necessárias à estimativa correta da técnica ou modelo: essa etapa inclui abordar, ilustrativamente, e guiar a implementação das correções aos vieses, deficiências e limitações discutidas na primeira parte do artigo.

5. Conclusões e diretrizes empíricas visando as boas práticas de pesquisa: por fim, os autores são convidados a oferecerem suas recomendações e diretrizes acerca de boas práticas de pesquisa relacionadas à utilização, por parte do pesquisadorleitor, do material disponibilizado ao longo do Artigo-Tutorial.

Figura 2. Estrutura ideal, porém não obrigatória, de um Artigo-Tutorial.

\section{COMO REVISAR UM ARTIGO-TUTORIAL?}

Todos os artigos submetidos à RAC passam obrigatoriamente pelo processo de revisão pelos pares de forma cega. O mesmo procedimento será utilizado na avaliação dos Artigos-Tutoriais. Ou seja, esses artigos também passarão obrigatoriamente pela revisão por pares de forma cega. Um dos principais questionamentos que temos recebido acerca do processo de submissão é como será o processo de revisão pelos pares.
Ou, de forma mais objetiva, como entendemos que nossos avaliadores deverão avaliar um ArtigoTutorial?

Inicialmente, é importante mencionar que, obrigatoriamente, os revisores de ArtigosTutoriais terão acesso à base de dados e aos códigos utilizados no artigo a ser avaliado. Naturalmente, os autores terão a prerrogativa de utilizar o pacote estatístico que preferirem e os avaliadores precisarão conhecer tal pacote utilizado pelos autores. O corpo editorial da 
RAC valerá de seus melhores esforços para que o avaliador seja especialista no pacote estatístico utilizado. Se, no entanto, houver a incapacidade de encontrar um avaliador especialista na ferramenta, o corpo editorial irá comunicar os autores sobre a inviabilidade de revisar o ArtigoTutorial e, portanto, de considera-lo à publicação.
Acreditamos, contudo, que não teremos problemas em encontrar revisores disponíveis a revisar códigos dos principais pacotes utilizados em pesquisa em Administração: R, SPSS, Stata, Matlab e Python. No que tange a avaliação do código do Artigo-Tutorial, oferecemos o seguinte checklist de avaliação (Fig. 3).

\section{Sobre o texto escrito referente ao Artigo-Tutorial:}

O Artigo-Tutorial é claro acerca do problema de pesquisa analisado e sobre sua relevância à pesquisa empírica em Administração? O Artigo-Tutorial apresenta o método ou modelo analisado de forma clara, objetiva e instrutiva?

A linguagem é clara, organizada, e compreensível mesmo por não-especialistas?

Os autores discutem profundamente o problema empírico em questão e oferecem didaticamente uma solução ao problema? O Artigo-Tutorial disponibiliza fácil acesso aos códigos e base e dados?

O Artigo-Tutorial descreve as operações mais importantes executadas pelo código (ou protocolo de pesquisa) e necessárias ao entendimento do método ou modelo analisado?

O Artigo-Tutorial descreve apropriadamente a base de dados e apresenta seus fatos estilizados mais importantes?

A solução, desdobramento ou discussão apresentada pelo Artigo-Tutorial é de interesse à comunidade de pesquisa em Administração?

O Artigo-Tutorial apresenta conclusões pertinentes e sugestões relevantes à aplicação futura do método ou técnica empírica analisada?

Sobre o código (ou protocolo de pesquisa) referente ao Artigo-Tutorial:

Os revisores conseguiram facilmente encontrar e fazer o download de todos os arquivos necessários à revisão do código? Os autores disponibilizaram um arquivo readme.txt com informações adicionais, se necessárias, relevantes à leitura e utilização do código?

O código é facilmente reproduzível e conduz o revisor diretamente às estatísticas e estimativas apresentadas no ArtigoTutorial?

O código é transparente e comunica com objetividade as suas escolhas empíricas feitas pelos autores?

As estatísticas e estimativas reportadas no Artigo-Tutorial são facilmente reproduzidas pelo código disponibilizado?

Os autores se valeram de pacotes disponibilizados diretamente pela ferramenta estatística utilizada?

Ao longo do código, os autores deixam claro se há necessidade de instalação de algum pacote oficial ou não-oficial para se obter as estimativas do Artigo-Tutorial?

Os autores se valeram do princípio da simplicidade nas escolhas empíricas acerca do código?

O código excluiu informações pessoais dos autores que permitam sua identificação?

Figura 3. Checklist de avaliação do texto e código do Artigo-Tutorial.

Oferecemos essa lista de itens que, sob nossos olhos, são relevantes à avaliação dos códigos pelos pares. Naturalmente, essa lista tem caráter de recomendação, de forma que os avaliadores terão sempre a prerrogativa de avaliar aqueles itens que julguem mais importantes. Ainda assim, recomendamos que os avaliadores a utilizem e que os autores levem seus itens em consideração no momento da submissão de um Artigo-Tutorial à RAC.

\section{AIMPORTÂNCIA DOS DADOS ABERTOS PARA O ENSINO E PESQUISA EM ADMINISTRAÇÃO}

Entendemos que o sucesso dessa nova seção da RAC passa pelas importantes vantagens que as práticas de Ciência Aberta oferecem aos pesquisadores e à comunidade científica. Em destaque, a disponibilização dos dados utilizados em pesquisas é indispensável para o sucesso na nova seção da RAC, mas também é fundamental para o futuro da pesquisa 
acadêmica em Administração. A RAC não está sozinha na sua iniciativa de aderir às práticas da Ciência Aberta. Estamos ao lado de diversas associações e periódicos internacionais de alto alcance e reconhecidos internacionalmente pela sua qualidade. Por exemplo:

\section{A American Economic Association requer} que

"Authors of accepted papers that contain empiricalwork, simulations, or experimental work must provide, prior to acceptance, information about the data, programs, and other details of the computations sufficient to permit replication, as well as information about access to data and programs." (American Economic Association, 2019, online).

\section{A Management Science requer que}

"Authors of accepted papers that contain numerical or computational work such as empirical or experimental studies, simulations, or numerical testing of algorithms or heuristics must provide, prior to the paper being sent to production, the data, programs, and other details of the experiment and computations sufficient to permit replication." (Informs, 2019, online).

\section{O Sociological Methods and Research} requer que

"Authors of quantitative empirical articles must make their data--along with all specialized computer programs, program recodes, and an explanatory file describing what is included and how to reproduce the published results--available for replication purposes." (SAGE Journals, n.d., online).

Essa lista no campo de periódicos, assumida como relevante para a área de negócios, pode facilmente continuar. Acreditamos que esse movimento, ao qual a RAC orgulhosamente se uniu, diminuirá os prejuízos da Dark Data (Petty, Stephenson and Hadley, 2019) e potencialmente diminuirá os efeitos negativos do viés de publicação (tradução livre para o termo em inglês positive publication bias) à nossa comunidade. Aos nossos olhos, ao se disponibilizar os dados, os códigos e todos materiais necessários à replicabilidade da pesquisa, o foco do pesquisador-leitor retorna à aplicação, ao rigor científico e ao método de pesquisa, ao invés de puramente aos resultados.
Em adição, a RAC entende que a pesquisa em Administração passa, de forma importante, pela capacidade de ensino de técnicas de pesquisa aos futuros pesquisadores. ArtigosTutoriais, além de apresentarem e se valerem do rigor científico necessário a uma pesquisa científica, tem a capacidade de tutoriar futuros pesquisadores acerca dos métodos analisados.

Como em diversas áreas correlatas à Administração, particularmente as que se valem de métodos qualitativos que ensejam subjetividade, os dados podem ser criados de forma contextual e subjetiva, e as análises potencialmente refletirão tal subjetividade. Essas características têm gerado reações diversas em relação a iniciativas de Ciência Aberta em pesquisas qualitativas (Chauvette, SchickMakaroff, \& Molzahn. 2019). Naturalmente, em Artigos-Tutoriais qualitativos, os autores terão sempre a prerrogativa de exercer seu senso de valor na utilização de uma determinada técnica de pesquisa, na interpretação contextual do fenômeno, na criação subjetiva de uma escala, etc.

Em paralelo, os revisores de ArtigosTutoriais qualitativos deverão ter o compromisso ético de exercer o julgamento transparente e não-viesado acerca dessas particularidades. Apesar de todas essas especificidades, entendemos que Artigos-Tutoriais qualitativos têm a potencialidade de exercer um importante papel educativo, na medida em que aumentam a transparência acerca de como os julgamentos de valores dos autores foram feitos, como o contexto foi interpretado, como o fenômeno foi observado, etc. Assim, a RAC convida e clama pela submissão de Artigos-Tutoriais que analisem técnicas qualitativas de pesquisa.

Finalmente, seguindo o compromisso de divulgar os trabalhos com dados abertos e de suportar seus autores, apresentamos a seguir a lista atualizada de artigos que, na data desse editorial, aderiram a práticas de Ciência Aberta. Agradecemos a esses autores por seu pioneirismo em suportar as iniciativas da RAC e em se unir ao movimento mundial por uma ciência mais aberta, inclusiva e democrática. 
Tabela 1. Artigos com dados abertos publicados na RAC desde 2018.

\begin{tabular}{|c|c|c|c|c|c|}
\hline \# & Título & Vol. & Nr. & Ano & Endereço eletrônico dos dados \\
\hline 1 & $\begin{array}{l}\text { Parliamentary Elections in Brazil: The Use of Twitter in the } \\
\text { Search for Votes }\end{array}$ & 22 & 4 & 2018 & http://dx.doi.org/10.17632/7b3vp6bz9p.1 \\
\hline 2 & $\begin{array}{l}\text { Cross Channel Consumer Behavior: A Moderate-Mediation } \\
\text { Model in Online/Offline Purchasing }\end{array}$ & 22 & 4 & 2018 & http://doi.org/10.5281/zenodo.1303670 \\
\hline 3 & Reasons and Intentions for Expatriation of Volleyball Players & 22 & 4 & 2018 & http://dx.doi.org/10.17632/d2jj7gdg82.1 \\
\hline 4 & $\begin{array}{l}\text { Strategic Information Systems Enabling Strategy-as-practice } \\
\text { Under Uncertain Environments }\end{array}$ & 22 & 4 & 2018 & http://doi.org/10.5281/zenodo.1304295 \\
\hline 5 & $\begin{array}{l}\text { Market logic as an efficiency measure of the organization of } \\
\text { Olinda's Carnival }\end{array}$ & 22 & 5 & 2018 & http://doi.org/10.5281/zenodo.1412806 \\
\hline 6 & Effectiveness of Crowdsourcing as a Support for Public Safety & 22 & 5 & 2018 & http://dx.doi.org/10.17632/73v2m9tn24.2 \\
\hline 7 & $\begin{array}{l}\text { Motivations and Consequences of Advisor Participation in } \\
\text { Mergers and Acquisitions }\end{array}$ & 22 & 6 & 2018 & http://dx.doi.org/10.17632/rfybj4xc6w.1 \\
\hline 8 & $\begin{array}{l}\text { Incongruent Humour, Advertising Effectiveness and Women: } \\
\text { An Experiment On Facebook }\end{array}$ & 23 & 1 & 2019 & http://dx.doi.org/10.17632/d6cgfnwt6r.1 \\
\hline 9 & $\begin{array}{l}\text { Newspaper Consumption in Print and Online: Printed } \\
\text { Newspapers is Status and Online is Easiness }\end{array}$ & 23 & 1 & 2019 & http://dx.doi.org/10.17632/cbw7rr72mn.1 \\
\hline 10 & $\begin{array}{l}\text { Where are We Heading? An Analysis of the Corporate } \\
\text { Governance Literature }\end{array}$ & 23 & 1 & 2019 & http://dx.doi.org/10.17632/fjn2cb9xnm.1 \\
\hline 11 & $\begin{array}{l}\text { Climate Strength: Its Role as a Moderator in the Relationship } \\
\text { Between Climate and Turnover }\end{array}$ & 23 & 1 & 2019 & http://dx.doi.org/10.17632/f3m2r2g994.1 \\
\hline 12 & $\begin{array}{l}\text { The Effects of Exploration, Exploitation, and Ambidexterity } \\
\text { on Software Firm Performance }\end{array}$ & 23 & 1 & 2019 & http://dx.doi.org/10.17632/tgcn93k4w5.1 \\
\hline 13 & $\begin{array}{l}\text { Effects of the Fleuriet model and liquidity ratios on tax } \\
\text { aggressiveness }\end{array}$ & 23 & 2 & 2019 & http://dx.doi.org/10.17632/m7df5tcf7t.1 \\
\hline 14 & $\begin{array}{l}\text { Academic Motivation Scale: Validity in Portuguese Public } \\
\text { Higher Education }\end{array}$ & 23 & 3 & 2019 & http://dx.doi.org/10.17632/6n78w5pz74.1 \\
\hline 15 & $\begin{array}{l}\text { The Impacts of Health Care Evaluations on the Well-Being of } \\
\text { Low-incomers }\end{array}$ & 23 & 3 & 2019 & http://dx.doi.org/10.17632/gn2jvckszx.1 \\
\hline 16 & $\begin{array}{l}\text { Effects of Qualitative Data Analysis Softwares in the Quality } \\
\text { of Researches }\end{array}$ & 23 & 3 & 2019 & http://dx.doi.org/10.17632/fcm3bmk7v5.1 \\
\hline 17 & $\begin{array}{l}\text { Formal and Informal Interpersonal Relationships: Interaction } \\
\text { of Networks in the Academic Environment }\end{array}$ & 23 & 3 & 2019 & http://dx.doi.org/10.17632/by5rhc9z9z.1 \\
\hline 18 & $\begin{array}{l}\text { Keep Innovating: Absorptive Capacity and the Performance } \\
\text { of Brazilian Information Technology Companies }\end{array}$ & 23 & 4 & 2019 & http://dx.doi.org/10.17632/3wtn38x5zw.1 \\
\hline 19 & $\begin{array}{l}\text { Are Incentives for Internationalization Appropriate? } \\
\text { Perception of Researchers in Information Systemss }\end{array}$ & 23 & 4 & 2019 & http://dx.doi.org/10.17632/3b529zn236.1 \\
\hline 20 & $\begin{array}{l}\text { Gender Diversity in Board of Directors and the Relationship } \\
\text { between Performance and Financial Risk in Family Firms }\end{array}$ & 23 & 6 & 2019 & http://doi.org/10.5281/zenodo.3445050 \\
\hline 21 & $\begin{array}{l}\text { Capital Structure and Governance Mechanisms External to } \\
\text { the Firm: A Cross-Country Analysis }\end{array}$ & 23 & 6 & 2019 & http://doi.org/10.5281/zenodo.3478652 \\
\hline 22 & $\begin{array}{l}\text { Expansion of Brazilian Federal Universities: Is It Possible to } \\
\text { Raise Economic Impacts? }\end{array}$ & 24 & 1 & 2020 & http://doi.org/10.17632/2c87cv2rw5.2 \\
\hline 23 & $\begin{array}{l}\text { Gift-giving, Love Styles, and Sacrifice: An Analysis Among } \\
\text { Romantic Partners }\end{array}$ & 24 & 1 & 2020 & http://dx.doi.org/10.17632/99ytkx8sr3.2 \\
\hline 24 & Work Engagement in the Public Service: A Multicultural Model & 24 & 1 & 2020 & http://dx.doi.org/10.17632/g857529zkw.3 \\
\hline 25 & $\begin{array}{l}\text { Political Cycles and Fiscal Management in Brazilian } \\
\text { Municipalities }\end{array}$ & 24 & 2 & 2020 & http://dx.doi.org/10.17632/zg7tvnrkn9.1 \\
\hline 26 & $\begin{array}{l}\text { Managers, Engagement and Political Behaviors: A Nonlinear } \\
\text { Relationship }\end{array}$ & 24 & 3 & 2020 & http://dx.doi.org/10.17632/syrw7xgynt.1 \\
\hline
\end{tabular}

Nota. A Revista de Administração Contemporânea (RAC) mantém centralizados os conjuntos de dados, materiais e códigos abertos publicados por este periódico em uma página do Harvard Dataverse (https://dataverse.harvard.edu/dataverse/rac/ recuperado em 11 de Fevereiro, 2020). 


\section{ESTA EDIÇÃO E NOVIDADES NA RAC EM 2020}

A RAC é uma revista pluralista e heterodoxa na área de pesquisa em gestão de negócios, preocupada principalmente com questões relativas a vínculos entre estratégia e gestão competitiva, além de fornecer resultados de pesquisas em sistemas e padrões, ferramentas de gerenciamento corporativo, organizações e gerenciamento, setores específicos da indústria, e respostas a questões contemporâneas, como desenvolvimento, resiliência das comunidades, desigualdade, consumo, tecnologia, e mudanças climáticas. A partir de uma perspectiva regional e espírito interdisciplinar, a RAC está posicionada na vanguarda da inovação teórica e metodológica, dando boas-vindas a contribuições substantivas e empíricas, que investigam e problematizam questões significativas de preocupação econômica, social e política, especialmente onde essas novas abordagens avançam.

Priorizamos o trabalho empiricamente engajado, e que promove abordagens epistemológicas críticas, que ampliem os limites conceituais, e coloque a teoria para atuar de maneiras inovadoras, e que navega conscientemente pelas políticas de produção de conhecimento, dentro e fora da academia. Os horizontes de administração contemporânea são amplos, mas os temas de preocupação recorrente para a Revista de Administração Contemporânea incluem tópicos sobre: Gestão ambiental, Produção, distribuição e consumo de recursos; Política urbana; Tecnologia, informação e conhecimento; Mercados financeiros, de capitais, e Finanças corporativas; Cadeia de suprimentos; Empreendedorismo; Mercados de trabalho; Comportamento organizacional e individual.

Desde dezembro de 2019, além de um layout totalmente renovado para acomodar o incremento de informações acerca dos documentos publicados, a RAC passou a adotar 3 práticas adicionais com intuito principal de consolidar a orientação da revista à transparência do processo editorial, assim como aumentar a audiência e a capilaridade do periódico. Primeiro, tomando como base o princípio da reciprocidade acadêmica (Mendes-Da-Silva, 2018b), passou a ser condição para submissão de manuscritos a disposição do autor que submeter o trabalho para atuar como revisor na RAC por até duas oportunidades em um período de 12 meses. Segundo, autores de manuscritos que forem aceitos, após o processo de avaliação via double blind review, devem fornecer uma versão em língua inglesa do trabalho aceito (se acaso a submissão já não tenha sido realizada na língua inglesa).

Terceiro, em linha com as práticas de ciência aberta, e a par do crescimento da comunidade de pesquisadores, mas mantendo o método double blind review (revisor e autor continuam anônimos entre si), a RAC passou a convidar os indivíduos revisores dos trabalhos aceitos para que esses voluntariamente aceitem que seus nomes sejam registrados nos respectivos trabalhos por eles avaliados. Com essa política, a RAC espera principalmente:

(I) colaborar para o reconhecimento do trabalho conduzido pelos avaliadores anônimos; (II) permitir que mais facilmente comunidades de pesquisadores interessados em determinados temas sejam identificadas; (III) incentivar que mais pesquisadores visionem benefícios decorrentes do trabalho inerente à avaliação de manuscritos submetidos; além dos demais benefícios líquidos da revisão pelos pares aberta (Martins, 2019; Mendes-Da-Silva, 2019a).

Tendo em vista que se mantendo o anonimato entre autores e avaliadores, até o possível aceite do trabalho para publicação, entende-se que permanecem preservadas as condições para o julgamento livre de vieses. Mas, por outro lado, adicionamos as vantagens decorrentes da transparência, tais como encorajar accountability e civilidade na comunicação entre autores e avaliadores, implicando em aumento da qualidade da revisão.

Como é de conhecimento da comunidade, todo processo editorial da RAC ocorre mediante a colaboração voluntária de colegas, desde autores, passando pelos avaliadores, até os editores. Nesta oportunidade registro um particular agradecimento ao Professor Victor Almeida (CoppeAd/UFRJ), pela competente liderança da Editoria de Casos para Ensino, entre junho/2018 e dezembro/2019. Para o exercício voluntário das atividades dessa Editoria foi convidada a Professora Paula Castro Pires de Souza Chimenti (CoppeAd/UFRJ), que prontamente aceitou o nosso convite. Boasvindas à Professora Paula, e esperamos que sua experiência na RAC seja de aprendizado e de contribuições para a comunidade. Aproveito o ensejo desta edição para fazer o honesto e sincero agradecimento aos indivíduos que, ao longo de 2019, atuaram voluntariamente como revisores na RAC. A nominata das mais de 200 pessoas segue nesta edição. 
Nesta edição, A RAC publica cinco artigos inéditos, sendo um deles com dados abertos. O primeiro trabalho, cujo título é 'Capacidade Relacional e Alianças Estratégicas de Pesquisa e Desenvolvimento', é de autoria de Taísa Scariot Preusler, Priscila Rezende da Costa, Tatiane Baseggio Crespi, e Geciane Silveira Porto. Conforme defendem os autores, o artigo tem como objetivo explorar como os processos da capacidade relacional podem contribuir para a geração de inovações, e por meio de uma abordagem qualitativa os autores defendem que a principal contribuição para o avanço do conhecimento foi um modelo interorganizacional para geração de inovações, a partir de alianças estratégicas de pesquisa e desenvolvimento, fundamentado nas evidências empíricas dos processos da capacidade relacional da Empresa Brasileira de Pesquisa Agropecuária (Embrapa), e dos seus parceiros externos.

$O$ segundo artigo (que possui dados abertos), de autoria de Romulo Matos de Moraes e Aridelmo José Campanharo Teixeira, tem como título 'Gestores, Engajamento e Comportamentos Políticos: Uma Relação Não Linear', registra como objetivo investigar como gestores, com diferentes níveis de Engajamento no Trabalho, encaram a presença de comportamentos políticos em suas organizações. Os autores defendem, como principal resultado, que na maioria dos casos analisados, a resiliência, o envolvimento, e a concentração mitigaram a percepção de comportamentos políticos na organização.

$O$ terceiro artigo é 'O "Problema da Imersão" nos Estudos do Empreendedorismo: Uma Proposição Teórica', e tem como autores Victor Silva Corrêa, Glaucia Maria Vasconcellos Vale, Pedro Lucas de Resende Melo e Marina de Almeida Cruz. Esse trabalho apresenta-se sob a forma de um ensaio teórico cujo objetivo é investigar o conceito de imersão e sua influência aos estudos do empreendedorismo. O trabalho apropria-se da literatura acerca de embeddedness de Granovetter, e associa-a a proposições clássicas da sociologia econômica de Karl Polanyi. Os autores chamam a atenção dos pesquisadores do empreendedorismo para a repercussão de outros tipos de imersão social. Ao mesmo tempo, sugere por meio da 'imersão total' a criação de novo modelo analítico, eventualmente capaz de ampliar as reflexões sobre as influências da imersão em diferentes estruturas à trajetória empreendedora. Finaliza com novas proposições, com destaque para abordagens e sugestões de investigações ainda hoje inexploradas.
O penúltimo artigo inédito desta edição é assinado por Gean Carlos Tomazzoni, Vânia Medianeira Flores Costa, Claudia Simone Antonello e Maria Beatriz Rodrigues, e tem como título 'Os Vínculos Organizacionais na Percepção de Gestores: Comprometimento, Entrincheiramento e Consentimento', como emprego de dados coletados junto a gestores de empresas do setor varejista localizadas em shopping centers, possui como objetivo analisar a percepção sobre os vínculos de comprometimento, entrincheiramento e consentimento do trabalhador com a organização, explorando seus fatores distintivos e seus significados para gestores. Conforme seus autores, este trabalho empírico aponta que as percepções dos gestores não só sustentam a delimitação conceitual dos modelos de comprometimento à sua base afetiva, mas também revelaram a saliência diferencial de outros vínculos organizacionais, como o entrincheiramento e o consentimento.

Por fim, o artigo de revisão intitulado 'Blockchain e a Perspectiva Tecnológica para a Administração Pública: Uma Revisão Sistemática', de autoria de Luzia Menegotto Frick de Moura, Daniela Francisco Brauner e Raquel JanissekMuniz, por meio de dados coletados em diferentes bases de dados: Scopus, Web of Science, SSRN e Science Direct, e seguindo o método PRISMA, apresenta potenciais aplicações e consequências do uso da Blockchain para a administração pública. Os autores defendem que as principais aplicações encontradas foram referentes a: processamento de dados e segurança de dados públicos, novas propostas de regulamentação estatal e de organização institucional. Quanto aos impactos, são enfatizadas: (I) a melhoria na gestão dos dados, (II) a diminuição da burocracia, e (III) necessidade de afinar a relação entre Estado, sociedade e mercado.

Este editorial realça que a RAC continua empenhada na missão e no compromisso de buscar maior contribuição deste periódico para a sociedade, e sobretudo para a comunidade de pesquisadores no campo de negócios. Os avanços alcançados nos últimos meses constituem as bases para a continuidade do trabalho de construir e manter o legado da RAC (Mendes-DaSilva, 2019b).

Convidamos todos à leitura deste número. 


\section{REFERÊNCIAS}

American Economic Association (2019). Data and code availability policy. Retrieved from https://www.aeaweb.org/journals/ policies/data-code/

Chauvette, A., Schick-Makaroff, K., \& Molzahn, A. E. (2019). Open data in qualitative research. International Journal of Qualitative Methods, 18, 1-6. https://doi.org/10.1177/1609406918823863

Elsevier (n.d.). New! "Tutorial articles" in Micron. Retrieved from https://www.journals.elsevier.com/micron/news/newtutorial-articles-in-micron

Informs (2019). Management science policy for data and code disclosure. Retrieved from https://pubsonline.informs. org/page/mnsc/datapolicy

Lanka, E., Lanka, S., Rostron, A., \& Singh, P. (2019). Research methods in qualitative management research. Zenodo. http://doi.org/10.5281/zenodo.3547828

Martins, H. (2019). Tutorial articles: Guiding the practice of quantitative research. Zenodo. http://doi.org/10.5281/zenodo.3558928

\section{Autores}

\section{Henrique Castro Martins*}

Rua Marquês de São Vicente, $\mathrm{n}^{\circ}$. 255, 22451-900, Rio de Janeiro, RJ, Brasil

E-mail: hcm@iag.puc-rio.br

(1) https://orcid.org/0000-0002-3186-4245

\section{Wesley Mendes-Da-Silva}

Rua Itapeva, 474, $8^{\circ}$ andar, 01332-000, São Paulo, SP, Brasil.

E-mail: rac.wesley.mendes@gmail.com

(1) https://orcid.org/0000-0002-5500-4872

* Autor Correspondente

\section{Corpo Editorial Científico e Equipe Editorial para esta edição:}

\section{Conselho Editorial}

Anielson Barbosa da Silva (UFPB, João Pessoa, Brasil)

Antonio Carlos Gastaud Maçada (UFRGS, Porto Alegre, Brasil)

Ely Laureano Paiva (FGV, São Paulo, Brasil)

Fabio Vizeu Ferreira (UP, Curitiba, Brasil)

Maria José Tonelli (FGV, São Paulo, Brasil)

Rogério Hermida Quintella (NPGA/UFBA, Salvador, Brasil)

Valmir Emil Hoffmann (UnB, Brasília, Brasil)

Wesley Mendes-da-Silva (EAESP/FGV, São Paulo, Brasil)

\section{Editor-chefe}

Wesley Mendes-da-Silva (EAESP/FGV, São Paulo, Brasil)

\section{Editores Associados}

André Luiz Maranhão de Souza-Leão (UFPE, Recife, Brasil)

Fabio Caldieraro (EAESP/FGV, São Paulo, Brasil)

Gilnei Luiz de Moura (UFSM, Santa Maria, Brasil)

Henrique Castro Martins (IAG PUC-Rio, Rio de Janeiro, Brasil)
Mendes-Da-Silva, W. (2018a). Promoção de transparência e impacto da pesquisa em negócios. Revista de Administração Contemporânea, 22(4), 639-649. https://doi.org/10.1590/1982-7849rac2018180210

Mendes-Da-Silva, W. (2018b). Reconhecimento da contribuição do avaliador anônimo. Revista de Administração Contemporânea, 22(5), 1-13. https://dx.doi.org/10.1590/1982-7849rac2018180281

Mendes-Da-Silva, W. (2019a). Revisão pelos Pares aberta e ciência aberta na comunidade de pesquisa em negócios. Revista de Administração Contemporânea, 23(4), 1-6. https://dx.doi.org/10.1590/1982-7849rac2019190278

Mendes-Da-Silva, W. (2019b). Relatório de gestão da Revista de Administração Contemporânea 2018-2019. Revista de Administração Contemporânea, 23(6). https://doi.org/10.1590/1982-7849rac2019190356

Petty, S., Stephenson, H., \& Hadley, S. (2019). Shining more light on dark data. Retrieved from https://www.csescienceeditor. org/article/shining-more-light-on-dark-data/

SAGE Journals (n.d). Sociological Methods \& Research publishing guidelines. Retrieved from https://journals.sagepub. com/author-instructions/SMR

Ismael Ali Ali (Kent State University, Ohio, EUA)

Marcus Cunha Junior (University of Georgia, EUA)

Paula Castro Pires de Souza Chiment (UFRJ/Coppead, Rio de Janeiro, Brasil)

Paulo César Matui (UniGranRio, Rio de Janeiro, Brasil)

Samy Dana (FGV/EAESP, São Paulo, Brasil)

\section{Corpo Editorial Científico}

Aureliano Angel Bressan (CEPEAD/UFMG, Belo Horizonte, Brasil)

Bryan Husted (York University, Canadá)

Carlos M. Rodriguez (Delaware State University, EUA)

Cristiana Cerqueira Leal (Universidade do Minho, Portugal)

Diógenes de Souza Bido (Mackenzie, São Paulo, Brasil)

Elin Merethe Oftedal (University of Tromsø, Noruega)

Emilio Jose Monteiro Arruda Filho (Unama, Belém, Brasil)

Fábio Frezatti (FEA/USP, São Paulo, Brasil)

Felipe Monteiro (Wharton/University of Pennsylvania, EUA)

Howard J. Rush (University of Brighton, Reino Unido)

James Robert Moon Junior (Georgia Institute of Technology, EUA)

John L. Campbell (University of Georgia, EUA)

José Antônio Puppim de Oliveira (United Nations University, Yokohama, Japão)

Julián Cárdenas (Freie Universität, Berlin, Alemanha)

Lucas Barros (EAESP/FGV, São Paulo, Brasil)

Luciano Rossoni (UniGranRio, Rio de Janeiro, Brasil)

M. Philippe Protin (Université Grenoble Alpes, França)

Paulo Estevão Cruvinel (Embrapa Instrumentação, São Carlos, Brasil)

Rodrigo Bandeira de Mello (Merrimack College, EUA)

Rodrigo Verdi (MIT Massachusetts Institute of Technology, Cambridge, EUA)

Valter Afonso Vieira, (UEM, Maringá, Brasil)

Wagner Kamakura (Jones Graduate School of Business, Rice University, Houston, EUA)

\section{Editoração}

Diagramação e Normas da APA: Kler Godoy (ANPAD, Maringá, Brasil)

Periodicidade: Bimestral.

Circulação: Acesso totalmente gratuito. 